\title{
Meeting the Need-from Institutional to Community Care
}

\author{
RHINEDD Toms, Consultant Psychiatrist, Severalls Hospital, Colchester
}

Severalls Hospital is a large psychiatric hospital, established in 1913 and originally designed for about 2000 patients. With the changes in attitudes to mental health over the last 70 years the hospital's aims and objectives have altered several times. Now, with the number of in-patients already reduced to approximately 650 and the movement to run down large hospitals such as this, preparation needs to be made for the shift to the community as the main future base for psychiatric services. Over the years the hospital has recognised the importance of preserving close links with the patient's home and has always maintained that regular activity and work is vital in the process of re-establishing patients in the community and in employment.

In spite of this, development of alternative community facilities for accommodation and treatment was slow, and after a visit by the Health Advisory Service in 1982 the comment was made that "rehabilitation services have not developed from their good historical base. The time has come for another thrust to catch up with what other Districts are doing". This series of articles aims to describe how that thrust was begun.

As in most other hospitals of this type, a large number of patients in the continuing care areas had been resident for many years. Many had no florid signs of psychiatric illness but were institutionalised and had largely lost the motivation and skills to look after themselves. These patients were accommodated in various wards and villas throughout the hospital, sometimes grouped together according to a common characteristic, e.g. high physical dependency or disturbed behaviour. Several consultants could admit patients to these wards and differing opinions on management and policy sometimes caused problems. In other wards patients under the care of one consultant were placed together regardless of level of function. There was no agreed system for placement of patients according to individual need. The Industrial and Oocupational Therapy Departments were responsible for a wide range of therapeutic activities, in particular the Household Management Unit and a house at the main hospital gate provided training in domestic chores and experience of independent living. Numbers which could be catered for were, however, small. Community accommodation for ex-psychiatric patients in Colchester and the surrounding district included a network of group homes run by a charitable organisation, a shortstay hostel for the younger age group run by Social Services, and a large number of boarding houses in nearby Clacton which housed not only ex-patients from Severalls but also those from other large psychiatric hospitals in the country. Total provision was, however, inadequate and a range of different types of staffed accommodation was badly needed, particularly in Colchester.
Money was made available for development of rehabilitation services, and a small multidisciplinary working group was established to examine ways in which a specialised system might be set up within the hospital. The initial proposal of this committee was that the whole of the continuing care area of the hospital would be included in the new rehabilitation system. The basic concept was that patients would be grouped according to their level of function and a preliminary survey identified several different groups. In spite of concerns not to create a 'two tier' system, with better services being provided for the more promising groups, it was decided that an efficient rehabilitation service must involve some degree of selection. Proposals were put forward for a small number of rehabilitation wards run by multidisciplinary teams and concentrating on intensive programmes for each individual in preparation for re-settlement in the community where possible. Responsibility for planning and setting up the rehabilitation services in the hospital and the community was passed to a new multidisciplinary rehabilitation steering group which has met weekly ever since.

With the fairly haphazard distribution of patients within the hospital, the identification of possible residents for the rehabilitation wards appeared to be a difficult problem. This was facilitated by the appointment of two principal psychologists with special responsibility for rehabilitation work. They organised a major survey of long-stay patients in the hospital, which included the gathering of personal information from each patient and scoring their day-to-day functioning in social and self-care activities on the $1983 \mathrm{Hall}$ \& Baker REHAB Rating Scale.

The first rehabilitation ward to be set up was Clare Court (an acronym from Community Living and Resettlement Environment), a 10 bedded ward which would concentrate on preparing patients from long-stay areas who wished to leave hospital for independent accommodation or group homes. An existing ward was upgraded and adapted to provide living and dining areas, pleasant bedrooms and a wellequipped kitchen. The nurse/patient ratio was high and an occupational therapist, domestic, and psychologist became members of the ward team. At a later date a consultant psychiatrist with a special interest in rehabilitation took over medical responsibility. Referrals to the ward were invited and each prospective resident was interviewed personally by members of the ward team before acceptance. Clare Court opened in May 1984 and a stay of about a year was envisaged for residents, although no strict limits were set. In early 1985 a three bedroomed house in the grounds, The Laurels, was made available to the rehabilitation system as a base for independent living experience before final discharge from hospital. In April 1985 three further 
rehabilitation wards were opened. Mistley, a 16 bedded integrated ward was designed for slower stream rehabilitation. Residents were thought to need 18 months to two years preparation for possible resettlement. Kent Ward ( 25 beds, male patients) and Kirby Ward ( 23 beds, female patients) contained mainly long-stay institutionalised patients who would not necessarily be resettled outside hospital. They could be expected to take part in a wider range of activities, take up opportunities to improve their quality of life, and start to re-use the skills of daily living.

Apart from the rehabilitation wards all other continuing care patients were relocated in wards appropriate to the level of functioning, each ward being under the care of one consultant psychiatrist.

Movement to and between the rehabilitation and longstay areas of the hospital is now controlled by a sub group of the rehabilitation steering group in order to maintain functional status and to avoid as far as possible increasing the long-stay hospital population. Referrals are considered weekly by the sub group and a preliminary assessment of the most suitable placement made. Each person is interviewed and work reports, REHAB scores, and opinions of existing ward staff are taken into account before a decision is made. Suggestions for alternatives to continuing hospital care are made where appropriate.

At present referrals to the rehabilitation system come from within the hospital only. Referral is made on a specific form and the referring ward staff are asked to fill in a REHAB Behaviour Rating Scale before the patient is interviewed by two members of the rehabilitation unit staff. On admission to one of the wards a detailed assessment procedure is carried out and strengths and weaknesses of that individual are noted. Up to four goal areas are then identified after discussion with the resident, e.g. personal hygiene, washing of clothes, cooking, etc. and progress, using the goal attainment scheme, is an important part of charting overall improvement. An individual programme is devised for each resident and regular multidisciplinary reviews of progress are held. Programmes may combine attendance at various industrial and occupational therapy areas of the hospital with activities on the ward, both social and domestic. Staff of the various departments-patients' employment bureau, industrial units, occupational therapy, social centre-work in close liaison with ward staff in organising residents' activities. No time limits are set for stay on any particular ward, but in general some degree of progress, however slow, is expected.

Volunteers are encouraged to become involved in the work of the rehabilitation unit. Two of the wards are already training areas for learner nurses and the other two intend to achieve similar status. Evaluation of the unit's work is made regularly by updating each resident's REHAB scores and a performance review produced by the ward every six months. Progress is being made in sharing activities between wards and departments and the monthly Rehabilitation Forum provides an opportunity for staff to meet regularly to discuss problems of organisation and management as well as clinical matters.

Although much of the work done so far in rehabilitation is still hospital based, the rehabilitation steering group is now concentrating on planning community facilities including both residential and day care provision. A property has been acquired for one further group home. A day centre and medium-stay hostel is being planned and financed jointly by Health and Social Services. It was felt that there was a great need for staffed permanent homes in the community for residents who could largely care for themselves but needed some degree of supervision. Operational policies for such 'community houses' are being drawn up and suitable properties being identified. There are also plans for converting former staff houses on the hospital site to supervised accommodation for a more elderly group of patients who because of age, length of stay, and vulnerability to change could not realistically be expected to live outside hospital. Other patients in this group may be accommodated in local authority elderly persons' homes. In this way we hope that a large number of the present residents of the rehabilitation wards can be placed in alternative facilities and cared for by community rehabilitation teams based in the two main areas of population, i.e. Colchester and Clacton.

It may be that hospital admission will still be required from time to time, but it seems unlikely that additions to the long-stay population will continue at the same rate as in the past. For the present the day hospital already existing at Severalls will need to be used for day activities for patients living in the community, but the setting up of facilities in Colchester town and in other areas is an urgent priority. These facilities need to cater for varying needs and may range from day centres to sheltered workshops.

The experience of setting up a new rehabilitation unit has been a stimulating and interesting process for all concerned. It would not have been possible but for the hard work and dedication of a very large number of people who have brought their individual skills and professional expertise to the task of working together as a multidisciplinary team. Now that the foundations have been laid we hope that the enthusiasm for further progress to community based care will continue.

Further articles in this series will deal with specific issues relating to work in the unit. Multidisciplinary team cooperation is described, with particular emphasis on the role of the key worker. The contribution of research is discussed in two articles, one dealing with a survey of long-stay patients within the hospital and the other with the needs in the community of people with long-term mental health problems.

This is the first of a series of four papers to be published in the Bulletin on rehabilitation developments at Severalls Hospital. 